\title{
Measuring Active-Sterile Neutrino Oscillations with a Stopped Pion Ne utrino Source
}

\author{
G.T. Garvey, A. Green, C. Green, W.C. Louis, G.B. Mills, \\ G. McGregor, H. Ray, R. Schirato, R.G. Van de Water, \\ D.H. White \\ Los Alamos National Laboratory \\ LA-UR-04-8716
}

October 6, 2018

\begin{abstract}
The possible existence of light sterile neutrinos is of great interest in many areas of particle physics, astrophysics, and cosmology. Furthermore, should the MiniBooNE experiment at Fermilab confirm the LSND oscillation signal, then new measurements are required to identify the mechanism responsible for these oscillations. Possibilities include sterile neutrinos, CP or CPT violation, variable mass neutrinos, Lorentz violation, and extra dimensions. In this paper, we consider an experiment at a stopped pion neutrino source to determine if activesterile neutrino oscillations with $\Delta \mathrm{m}^{2}$ greater than $0.1 \mathrm{eV}^{2}$ can account for the signal. By exploiting stopped $\pi^{+}$decay to produce a monoenergetic $\nu_{\mu}$ source, and measuring the rate of the neutral current reaction $\nu_{\mathrm{x}}{ }^{12} \mathrm{C} \rightarrow \nu_{\mathrm{x}}{ }^{12} \mathrm{C}^{*}(15.11)$ as a function of distance from the source, we show that a convincing test for active-sterile neutrino oscillations can be performed.
\end{abstract}

\section{Introduction}

The understanding of neutrino mass has undergone a revolution over the last ten years. Neutrino mass, via oscillations, is now experimentally established [1]. The Standard Model may now need to accommodate right handed neutrinos, $\nu_{R}$, that are sterile with respect to the weak 
interaction. The new issue to be addressed concerns the mass spectrum of these sterile neutrinos. Although many theorists postulate that $\nu_{R}$ are heavy via the see-saw mechanism [2], there is no experimental evidence for the mass spectrum of sterile neutrinos or for the number of generations [3. In fact, light sterile neutrinos have been invoked to explain many puzzles, such as the LSND result [4, the solar neutrino energy spectrum anomaly [5], the R-process in type II supernovae [6], pulsar kicks [7], dark matter [8], the perplexing dark energy [9], and most recently, extra dimensions [10]. Thus, much experimental and theoretical justification exists to motivate a search for light sterile neutrinos above the mass of $0.1 \mathrm{eV}^{2}$.

The LSND result points to active-sterile neutrino oscillations in the $\Delta \mathrm{m}^{2}$ range of 0.1 to $2 \mathrm{eV}^{2}$ [11. A recent analysis of a $(3+2)$ sterile neutrino model indicates the possibility of a second sterile neutrino solution around $20 \mathrm{eV}^{2}$ 4]. In addition, a supernovae R-process analysis favors a $3<\Delta \mathrm{m}^{2}<70 \mathrm{eV}^{2}$ sterile neutrino [6]. We propose an experiment that is capable of the direct detection of active-sterile neutrino oscillations in this mass region. If we interpret the LSND result as an intermediate sterile state, i.e. $\bar{\nu}_{\mu} \rightarrow \bar{\nu}_{\mathrm{s}} \rightarrow \bar{\nu}_{\mathrm{e}}$, where the observed LSND oscillation probability is $P\left(\bar{\nu}_{\mu} \rightarrow \bar{\nu}_{\mathrm{e}}\right)=(0.264 \pm 0.081) \%$ [11], then coupling this with short baseline reactor experimental limits on $\nu_{\mathrm{e}}$ disappearance of $P\left(\bar{\nu}_{\mathrm{e}} \rightarrow \bar{\nu}_{\mathrm{s}}\right)<10 \%$ [12], we can predict the $\nu_{\mu}$ disappearance to sterile neutrinos to be $P\left(\bar{\nu}_{\mu} \rightarrow \bar{\nu}_{\mathrm{s}}\right)>10 \%$. The above arguments set the scale for the $\Delta \mathrm{m}^{2}$ and $\sin ^{2} 2 \theta$ sensitivity that needs to be achieved.

\section{Beyond the Standard Model}

Given the prospect of a new intense stopped pion neutrino source, together with a positive signal from MiniBooNE [13, a next generation short baseline neutrino experiment needs to be carefully considered. A neutrino detector at the Spallation Neutron Source (SNS) has already been discussed [14 that demonstrates how an improved measurement in the LSND/MiniBooNE region can be made. Improving the $\Delta \mathrm{m}^{2}$ and $\sin ^{2} 2 \theta$ measurement of any positive signal from MiniBooNE would be important. However, the nagging question would still remain as to the new physics behind the three distinct $\Delta \mathrm{m}^{2}$ regions that have been measured with solar, reactor, atmospheric, and short baseline detectors. Several possibilities at present exist, such as sterile neutrinos [4], CP or CPT violation [15], mass varying neutrinos 9], Lorentz violation [16], and extra dimensions [10].

The CP or CPT violation possibility could be readily tested with a MiniBooNE/LSND style detector coupled with a short duty-factor stopped pion beam which allows, via timing cuts, a separation of $\nu_{\mu}$ and $\bar{\nu}_{\mu}$ events. Thus, a direct comparison of oscillation probabilities from these two simultaneous data sets will allow a test of CPT and CP violation models. Mass 
varying neutrinos can be tested by changing the density of material between the source and detector. The Lorentz violation model can be tested by looking for sidereal variations in the neutrino flux. These three tests will be discussed in more detail in a future paper.

This paper discusses the first possibility, that of sterile neutrinos. To demonstrate activesterile neutrino oscillations directly, and achieve the best sensitivity, requires a two detector setup and a stopped pion neutrino source. The following sections describe how this can be done.

\section{Intense Stopped Pion Neutrino Sources}

To detect sterile neutrino effects in the mass range of LSND and above requires an intense source of well characterized neutrinos. Stopped pion decay from a low energy proton beam is such a source. Neutrinos from stopped pion decay have a well defined flux, well defined energy spectrum, and low backgrounds. Fortunately, accelerators that can provide such a source are either being built or are being proposed. The first such accelerator is the $1.4 \mathrm{MW}, 1.3 \mathrm{GeV}$, short duty-factor Spallation Neutron Source (SNS) [17, which is currently being built and will be fully commissioned by 2008. The second is a proposal for a $2 \mathrm{MW}, 8 \mathrm{GeV}$, Proton Driver (PD) at FNAL [18.

The dominant decay scheme that produces neutrinos from a stopped pion source is

$$
\pi^{+} \rightarrow \mu^{+} \nu_{\mu}, \quad \tau=26 \mathrm{nsec}
$$

followed by

$$
\mu^{+} \rightarrow e^{+} \bar{\nu}_{\mu} \nu_{e}, \quad \tau=2.2 \mu \text { sec. }
$$

The neutrinos from stopped $\pi^{-}$'s are highly suppressed because the negative pions are absorbed in the surrounding material. Thus, neutrinos from the $\pi^{-}$decay chain are significantly depleted and can be estimated from the measured $\nu_{\mu}, \bar{\nu}_{\mu}$, and $\nu_{\mathrm{e}}$ flux. Figure 1 shows the neutrino time and energy spectra from a stopped pion source. As shown in the right hand plot, the $\nu_{\mu}$ energy is monoenergetic $\left(E_{\nu_{\mu}}=29.8 \mathrm{MeV}\right)$, while the $\bar{\nu}_{\mu}$ and $\nu_{\mathrm{e}}$ have known Michel decay distributions with an end point energy of $52.8 \mathrm{MeV}$. Furthermore, the left hand plot shows the SNS beam timing and the time distributions for the three neutrino species. One of the advantages of the SNS relative to the LAMPF accelerator is the three orders of magnitude shorter beam time of $695 \mathrm{nsec}$ (the FNAL PD will have a similar beam time). With a simple beam-on timing cut, one can obtain a fairly pure $\nu_{\mu}$ sample, with only a $14 \%$ contamination of $\bar{\nu}_{\mu}$ and $\nu_{\mathrm{e}}$ each. This remaining background is easily measured and subtracted. 

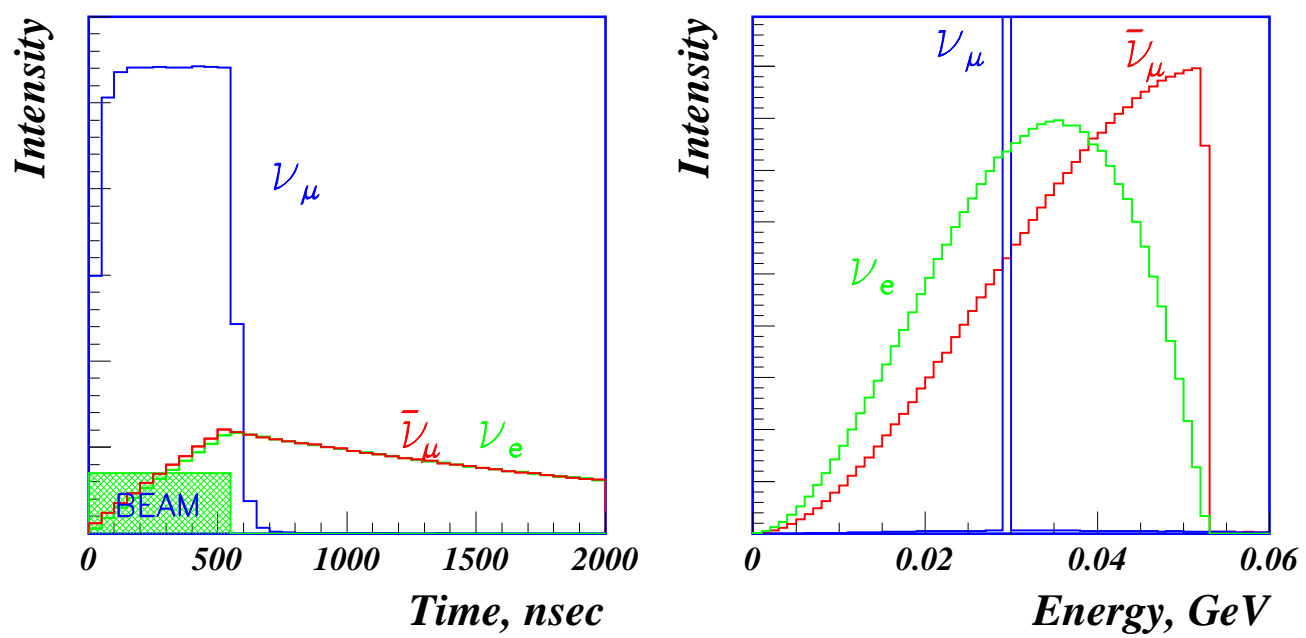

Figure 1: The neutrino time and energy spectra of the different neutrino species produced isotropically from a stopped pion source [17].

\begin{tabular}{|c|c|c|c|}
\hline & FNAL $(8 \mathrm{GeV})$ & FNAL $(5 \mathrm{GeV})$ & SNS \\
\hline $\mathrm{P} / \mathrm{yr}$ & $1.6 \times 10^{22}$ & $1.6 \times 10^{22}$ & $6.7 \times 10^{22}$ \\
\hline $\mathrm{DAR} \nu(\nu / P)$ & 1.5 & 0.9 & 0.13 \\
$\mathrm{DAR} \nu(\nu / y r)$ & $7.3 \times 10^{22}$ & $4.4 \times 10^{22}$ & $2.9 \times 10^{22}$ \\
\hline
\end{tabular}

Table 1: Proton intensities at FNAL and SNS. The numbers are taken from [18, assuming $3.15 \times 10^{7} \mathrm{~s} / \mathrm{yr}$ operation.

Table 1 shows the expected proton rates for both SNS and FNAL beamlines, normalized to a full year of running, i.e. $3.15 \times 10^{7}$ seconds. The FNAL Proton Driver proposal is broken down into $8 \mathrm{GeV}, 2 \mathrm{MW}$ and $5 \mathrm{GeV}, 1.25 \mathrm{MW}$ beamlines. The FNAL $8 \mathrm{GeV}$ option will provide about 2.5 times more protons per year than the SNS. However, the FNAL PD is only a proposal, while the SNS is under construction and will be operational by 2008. This makes the SNS a more timely option. Furthermore, the SNS is planning for 2014 an upgrade which will deliver 3 MW to two sources, making the interesting situation of multiple baselines with a single detector.

A key component of the sterile neutrino measurement is the physical size of the stopped pion source, which adds an uncertainty to the neutrino path length. For the SNS, the compact liquid mercury target will contribute approximately $25 \mathrm{~cm}$ (FWHM) to the neutrino path length uncertainty. The FNAL source size should be of similar dimensions to minimize neutrino path length uncertainties. 


\section{Active-Sterile Neutrino Oscillation Measurements}

The direct observation of active-sterile neutrino oscillations with $\Delta \mathrm{m}^{2}>0.1 \mathrm{eV}^{2}$ can be achieved because of two key features of a short duty-factor stopped pion neutrino source. Firstly, all active neutrino species $\left(\mathrm{x}=\nu_{\mathrm{e}}, \nu_{\mu}, \nu_{\tau}\right)$ can be efficiently measured using the superallowed neutral current reaction $\nu_{\mathrm{x}}{ }^{12} \mathrm{C} \rightarrow \nu_{\mathrm{x}}{ }^{12} \mathrm{C}^{*}(15.11)$ [19], where the $\mathrm{C}^{*}(15.11)$ state has a $92 \pm 2 \%$ branching fraction to the ground state [20]. A deficit of the neutral current rate in ${ }^{12} \mathrm{C}$ from expectation can only be from oscillations to sterile neutrinos. Secondly, using beam timing cuts, we can extract a high purity sample of $29.8 \mathrm{MeV}$ monoenergetic $\nu_{\mu}$ events. In $(3+1)$ models with $\Delta \mathrm{m}^{2}>0.1 \mathrm{eV}^{2}$, active-sterile oscillations can be approximated by two neutrino mixing. The oscillation probability can be expressed as:

$$
P\left(\nu_{\mu} \rightarrow \nu_{\mu}\right)=1-\sin ^{2} 2 \theta \sin ^{2}\left(1.27 \Delta \mathrm{m}^{2} \frac{\mathrm{L}}{29.8}\right) .
$$

Given these two features, we can observe active-sterile neutrino oscillations by measuring changes in the $\nu_{\mathrm{x}}{ }^{12} \mathrm{C} \rightarrow \nu_{\mathrm{x}}{ }^{12} \mathrm{C}^{*}(15.11)$ rate as a function of $\mathrm{L}$ (in meters). Figure 2 shows the oscillation length as a function of $\Delta \mathrm{m}^{2}$. Thus, a two detector configuration of the appropriate size and distance can achieve the desired sensitivity. A near detector greater than three meters in length and about 10-20 meters from the source will be sensitive to $\Delta \mathrm{m}^{2}>20 \mathrm{eV}^{2}$. A second large detector (required to increase statistics because of decreasing $1 / \mathrm{r}^{2}$ neutrino flux) at 50-100 meters will be sensitive to $\Delta \mathrm{m}^{2}>0.1 \mathrm{eV}^{2}$. Figure 3 shows a simple schematic of a detector setup that would allow sensitivity to sterile neutrino oscillations using the $\nu_{\mathrm{x}}{ }^{12} \mathrm{C} \rightarrow$ $\nu_{\mathrm{x}}{ }^{12} \mathrm{C}^{*}(15.11)$ channel.

A possible detector configuration at the SNS would be first a 25 ton fiducial mass $(3.5 \mathrm{~m}$ on a side) liquid scintillator near detector at $18 \mathrm{~m}$ distance from the neutrino source. This is the homogeneous detector proposed by the $\nu$-SNS collaboration to measure neutrino carbon cross sections [17]. The limited fiducial mass size of the near detector might be improved with future developments in flat PMT technology. Next would be a 500 ton fiducial mass liquid scintillator (6 $\mathrm{m}$ radius) far detector at $60 \mathrm{~m}$ distance from the neutrino source. Both designs would be similar to an LSND style detector, i.e. a mineral oil detector doped with butyl PBD scintillator to increase light output, instrumented with $25 \%$ photocathode coverage. This gives approximately a $7 \%$ energy resolution for reconstruction of the $15.11 \mathrm{MeV}$ gamma ray. Both detector masses are quoted in fiducial mass, which is the useful mass available for reliable event reconstruction. To estimate NC neutrino rates in the near and far detector, we scale to the detection rate calculations in [14, which have assumed a $\nu_{\mu}{ }^{12} \mathrm{C} \rightarrow \nu_{\mu}{ }^{12} \mathrm{C}^{*}(15.11)$ cross section of $2.7 \times 10^{-42} \mathrm{~cm}^{2}[19$. The Karmen collaboration has measured this cross section 


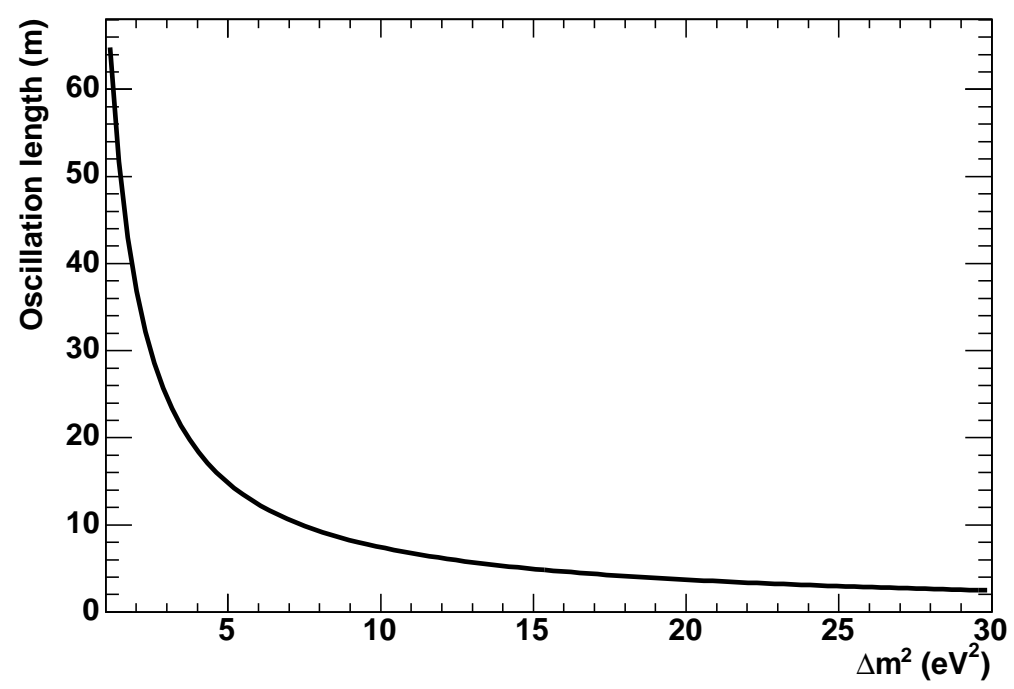

Figure 2: Oscillation length as a function $\Delta \mathrm{m}^{2}$ where $E_{\nu_{\mu}}=29.8 \mathrm{MeV}$.

\section{Neutral Current Disappearance Pattern in a Two Detector Setup}

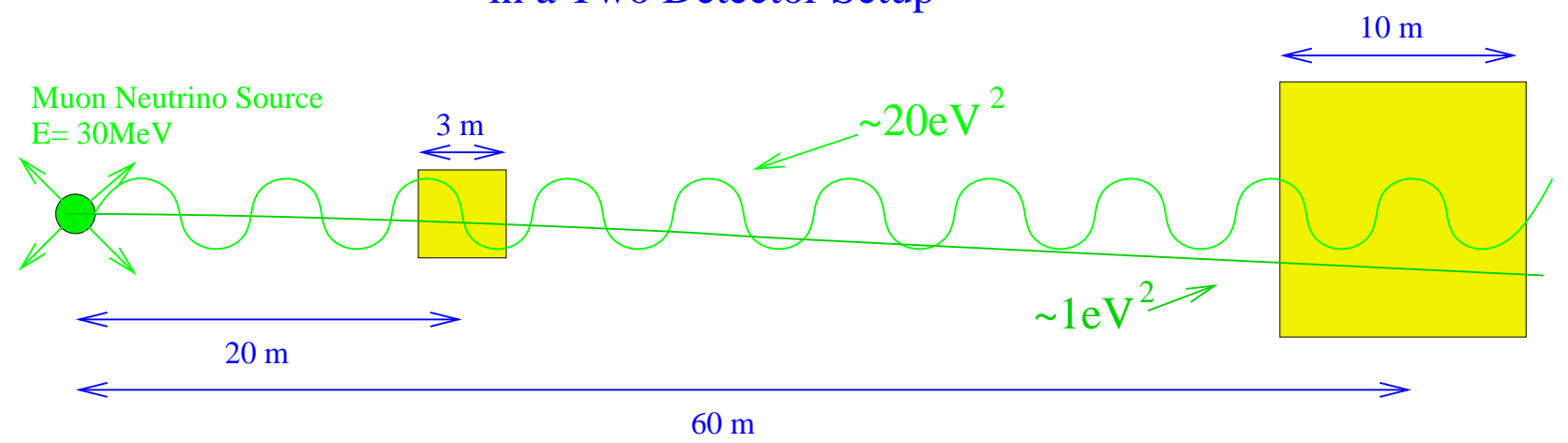

Figure 3: Neutral Current $\nu_{\mathrm{x}}{ }^{12} \mathrm{C} \rightarrow \nu_{\mathrm{x}}{ }^{12} \mathrm{C}^{*}(15.11)$ disappearance patterns for a two detector setup. 


\begin{tabular}{|c|c|c|c|c|}
\hline Detector & Source Dist. (m) & FD Size (tons) & FD Length $(\mathrm{m})$ & $\nu_{\mu}{ }^{12} C \rightarrow \nu_{\mu}{ }^{12} C^{*}$ events $/$ yr \\
\hline SNS Near & 18 & 25 & 3 & 2056 \\
SNS Far & 60 & 500 & 10 & 3701 \\
\hline FNAL Near & 10 & 116 & 5 & 77806 \\
FNAL Far & 100 & 1300 & 12 & 8720 \\
\hline
\end{tabular}

Table 2: Estimated $\nu_{\mu}{ }^{12} \mathrm{C} \rightarrow \nu_{\mu}{ }^{12} \mathrm{C}^{*}(15.11)$ events per year at the SNS (1.4 MW) and FNAL (2 MW) sources, assuming $100 \%$ event reconstruction efficiency.

with limited statistics, getting a value of $\left(3.2 \pm 0.5_{\text {stat }} \pm 0.4_{\text {syst }}\right) \times 10^{-42} \mathrm{~cm}^{2}$ [21], consistent with the calculated cross section.

For a proposed experiment at FNAL, we would not have space restrictions, allowing for larger near and far detectors. We assume a longer baseline, out to $100 \mathrm{~m}$, and a detector mass of 2 ktons ( $8 \mathrm{~m}$ radius), which would be required to keep roughly the same statistics. This would give a fiducial mass of $1.3 \mathrm{ktons}$ ( $7 \mathrm{~m}$ radius).

Table 2 shows the number of events expected per year for the $\nu_{\mathrm{x}}{ }^{12} \mathrm{C} \rightarrow \nu_{\mathrm{x}}{ }^{12} \mathrm{C}^{*}(15.11)$ reaction for the two possible configurations, one at SNS with 1.4 MW, and the other at FNAL with $2 \mathrm{MW}$. The event reconstruction efficiency was assumed to be $100 \%$, as the $15.11 \mathrm{MeV}$ gamma ray is relatively easy to detect.

A Monte Carlo calculation was performed to estimate the $\nu_{\mathrm{x}}{ }^{12} \mathrm{C} \rightarrow \nu_{\mathrm{x}}{ }^{12} \mathrm{C}^{*}(15.11)$ oscillation sensitivity, which includes smearing of $25 \mathrm{~cm}$ (FWHM) from the finite neutrino source size and $50 \mathrm{~cm}$ (FWHM) from the reconstructed position resolution for the $15.11 \mathrm{MeV}$ gamma ray. Also included is background subtraction of charged current (CC) and neutral current (NC) events from $\bar{\nu}_{\mu}$ and $\nu_{\mathrm{e}}$ in the beam window [14. Because we can isolate a clean sample of these backgrounds in the time window after the beam, we will have an excellent estimation of their number and character, e.g. for the SNS far detector this is about 1300 events per year. This background increases the statistical error on the measured $\nu_{\mu}{ }^{12} \mathrm{C} \rightarrow \nu_{\mu}{ }^{12} \mathrm{C}^{*}(15.11)$ rate by at most $45 \%$. The background contribution from cosmic rays is negligible because of the short duty factor of the beam.

Figures 4 and 5 show the $(3+1)$ active-sterile neutrino oscillation sensitivity for a three year run at the SNS and FNAL proton driver with two detectors and a $5 \%$ normalization systematic errors. Both setups achieve a $\nu_{\mu} \rightarrow \nu_{\mathrm{s}}$ oscillation sensitivity of $3 \sigma$ for $\Delta \mathrm{m}^{2}>0.4 \mathrm{eV}^{2}$ and $\sin ^{2} 2 \theta>0.05$. This sensitivity is desired if the LSND signal is due to active-sterile neutrino oscillations.

The oscillation sensitivity plots are generated from a simultaneous fit to both the L shape 
and to an overall event normalization. The L measurement systematic errors are discussed above. The event normalization requires knowledge of the $\nu_{\mu}$ flux times neutral current cross section $\left(\phi_{\nu_{\mu}} \times \sigma_{\mathrm{NC}}\right)$. From rate calculations alone, we expect systematic errors on the order of $10 \%$, which is what LSND was able to achieve [11. Another technique is to use the charged current $\nu_{\mathrm{e}}{ }^{12} \mathrm{C} \rightarrow \mathrm{e}^{-12} \mathrm{~N}_{\mathrm{gs}}$ reaction to normalize the $\mathrm{NC}$ rate, as the final state $\mathrm{A}=12$ nuclear form factors are isotopic analogues, leading to systematic errors in the $\mathrm{NC} / \mathrm{CC}$ ratio of $\sim$ $3 \%$ 21]. Here we assume there are no oscillations of $\nu_{\mathrm{e}}$. The $\mathrm{NC} / \mathrm{CC}$ ratio is dominated by systematic errors since the event rates for both reactions are large. Besides the theoretical ratio error (discussed above), we need to know the relative fiducial volume efficiency and the relative detection efficiency of the $15.11 \mathrm{MeV} \gamma$ to that of the CC electron plus ${ }^{12} \mathrm{~N}$ beta decay. Based on experiences at LSND, these two errors are conservatively estimated at $3 \%$ each. This gives a total systematic uncertainly in the NC/CC ratio of $5 \%$, which translates into a $\nu_{\mu}{ }^{12} \mathrm{C} \rightarrow \nu_{\mu}{ }^{12} \mathrm{C}^{*}(15.11)$ event rate $\phi_{\nu_{\mu}} \times \sigma_{\mathrm{NC}}$ systematic error of $5 \%$.

If we consider just a single detector setup at the SNS, either near or far, Figures 6 and 1 show what might be expected for $5 \% \phi_{\nu_{\mu}} \times \sigma_{\mathrm{NC}}$ errors. Figure 6 shows that even this one near detector has good sensitivity to active-sterile oscillations with parameters of $\Delta \mathrm{m}^{2}>1 \mathrm{eV}^{2}$ and $\sin ^{2} 2 \theta>0.10$ at $3 \sigma$. This interesting measurement can be performed in parallel to the neutrino carbon cross section measurement planned for the $\nu$-SNS detector [17].

In figures 4 through 9 , two black stars are plotted that correspond to the $(3+2)$ model sterile neutrino solution $\Delta \mathrm{m}_{41}^{2}=0.9 \mathrm{eV}^{2}, \sin ^{2} 2 \theta_{41}=0.15, \Delta \mathrm{m}_{51}^{2}=22 \mathrm{eV}^{2}, \sin ^{2} 2 \theta_{51}=0.19$ [4]. This $(3+2)$ model gives significantly better fits than $(3+1)$ models 4. Although the sensitivity curves are strictly for $(3+1)$ models and cannot be directly applied to the $(3+2)$ solutions, it can be seen that all beam/detector combinations will be sensitive to these solutions. It should be noted that the favored $(3+1)$ solution from [4] is very close to the lower $\Delta \mathrm{m}^{2}(3+2)$ solution, and so this solution will have coverage at the $3 \sigma$ to $5 \sigma$ level. A recent $(3+1)$ analysis of all the short baseline data, excluding LSND, gives $\nu_{\mu} \rightarrow \nu_{\mathrm{S}}$ oscillation plots that exclude the upper right regions shown here for $\Delta \mathrm{m}^{2}>1 \mathrm{eV}^{2}$ and $\sin ^{2} 2 \theta>0.1$ at the $99 \%$ level [22]. Hence, even ignoring LSND, there is much untested phase space that can be probed for active-sterile neutrino oscillations.

Explicit treatment of $(3+2)$ models is shown in figures 8 and 9 where we present parameter determination of the $(3+2)$ solutions. These models cannot be approximated by a two neutrino approximation, and consequently $\sin ^{2} 2 \theta$ can no longer be interpreted as before. We instead define $\sin ^{2} 2 \theta$ as $4 \mathrm{U}_{\mu n}^{2}\left(1-\mathrm{U}_{\mu n}^{2}\right)$, where $\mathrm{U}_{\mu n}$ is the relevant element in the neutrino mixing matrix and $n$ is the sterile generation label. In these figures, one of the two solutions is assumed and fixed. This is a reasonable procedure since the two solutions have quite different 
characteristics, i.e. the low $\Delta \mathrm{m}^{2}$ solution is demonstrated by a rate suppression between two detectors, while the high $\Delta \mathrm{m}^{2}$ solution is demonstrated by rapid wiggles in L. This allows for easy separation of the two solutions. The resulting plot, therefore, shows the parameter determination at a single point in the full four dimensional parameter space, rather than a projection in this space. Both figures show that the parameter determination is excellent.

\section{Conclusion}

In the coming year, new physics beyond the Standard Model will be implied if MiniBooNE confirms the LSND oscillation anomaly. To understand whether active-sterile neutrino oscillations are responsible for this new physics will require a new class of oscillation experiments. A stopped pion source such as the SNS, coupled with two well positioned large liquid scintillator detectors, can achieve the desired sensitivity. This is made possible by the fortuitous combination of a monoenergetic $\nu_{\mu}$ flux and the efficiently reconstructed neutral current interaction $\nu_{\mathrm{x}}{ }^{12} \mathrm{C} \rightarrow \nu_{\mathrm{x}}{ }^{12} \mathrm{C}^{*}(15.11)$. The SNS two detector setup with $5 \% \phi_{\nu_{\mu}} \times \sigma_{\mathrm{NC}}$ systematic errors can explore the $\nu_{\mu} \rightarrow \nu_{\mathrm{s}}$ oscillation region $\Delta \mathrm{m}^{2}>0.4 \mathrm{eV}^{2}$ and $\sin ^{2} 2 \theta>0.05$ at the $3 \sigma$ level. This would cover the $\nu_{\mu} \rightarrow \nu_{\mathrm{S}}$ oscillation solutions indicated by LSND and the $(3+2)$ model. Such a measurement would be the first clear indication of the existence of light sterile neutrinos, and would herald a new era in neutrino physics.

\section{Acknowledgements}

We thank Yuri Efremenko, Tony Gabriel, and Michel Sorel for helpful discussions. This research was supported by LDRD funding at LANL.

\section{References}

[1] Y. Fukuda et al. [Super-Kamiokande Collaboration], Phys. Rev. Lett. 81, 1562 (1998) arXiv:hep-ex/9807003; Q. R. Ahmad et al. [SNO Collaboration], Phys. Rev. Lett. 89, 011301 (2002) arXiv:nucl-ex/0204008; K. Eguchi et al. [KamlAND Collaboration], Phys. Rev. Lett. 90, 021802 (2003) arXiv:hep-ex/0212021.

[2] M. Gell-Mann, P. Ramond, R. Slansky, in SuperGravity, ed. by P. Van Nieuwenhuizen, D.Z. Freedman (North Holland, Amsterdam 1979), p. 315. But see, for example, G. J. 
Stephenson, Jr. T. Goldman, B. H. J. McKellar and M. Garbutt, hep-ph/0404015 A. de Gouvea, hep-ph/0501039.

[3] A.Yu. Smirnov, arXiv:hep-ph/0411194

[4] M. Sorel, J.M. Conrad, M.H. Shaevitz, arXiv:hep-ph/0305255.

[5] P.C. de Holanda, A.Yu. Smirnov, arXiv:hep-ph/0307266.

[6] A.B. Balantekin, G.M. Fuller, arXiv:astro-ph/0309519 G. C. McLaughlin, J. M. Fetter, A. B. Balantekin and G. M. Fuller, Phys. Rev. C 59, 2873 (1999) arXiv:astro-ph/9902106.

[7] G.M. Fuller, A. Kusenko, I. Mocioiu, S. Pascoli, arXiv:astro-ph/0307267.

[8] K. Abazajian, G. M. Fuller and M. Patel, Phys. Rev. D 64, 023501 (2001) arXiv:astro-ph/0101524.

[9] D.B. Kaplan, A.E. Nelson, N. Weiner, arXiv:hep-ph/0401099. R. Fardon, A. E. Nelson and N. Weiner, JCAP 0410, 005 (2004); P. Q. Hung, hep-ph/0010126 P. Q. Hung, H. Pas, astro-ph/0311131 G. J. Stephenson, Jr. T. Goldman and B. H. J. McKellar, Int. J. Mod. Phys. A13, 2765 (1998) hep-ph/9603392; P. Gu, X. Wang and X. Zhang, Phys. Rev. D 68, 087301 (2003) arXiv:hep-ph/0307148.

[10] H. Pas, S. Pakvasa and T. J. Weiler, arXiv:hep-ph/0504096.

[11] A. Aguilar et al. [LSND Collaboration], Phys. Rev. D 64, 112007 (2001) arXiv:hep-ex/0104049.

[12] Felix Boehm, nucl-ex/9906010.

[13] A. O. Bazarko [BooNe Collaboration], arXiv:hep-ex/9906003

[14] G.J. VanDalen, nucl-ex/0309014.

[15] G. Barenboim, L. Borissov, J. Lykken, hep-ph/0212116 V. Barger, M. Sorel, and K. Whisnant, in preparation.

[16] V. A. Kostelecky, M. Mewes, arXiv:hep-ph/0406255.

[17] The Spallation Neutron Source (SNS) is an accelerator-based source being built in Oak Ridge, Tennessee, by the U.S. DOE, http://sns.gov/ Also see http://www.phy.ornl.gov/workshops/sns2/ for details on the neutrino source and cross section detector $\nu$-SNS.

[18] S.J. Brice, S. Geer, K. Paul, R. Tayloe, arXiv:hep-ex/0408135.

[19] M. Fukugita, Y. Kohyama and K. Kubodera, Phys. Lett. B 212, 139 (1988). 
[20] G. Baym, et al. Nuclear Physics (NUPABL), Volume A433 (1985), No. 1, pg 61.

[21] B. Armbruster [KARMEN Collaboration], Phys. Lett. B 423 (1998), 15-20.

[22] M. Cirelli, G. Marandella, A. Strumia and F. Vissani, Nucl. Phys. B 708, 215 (2005) arXiv:hep-ph/0403158. 


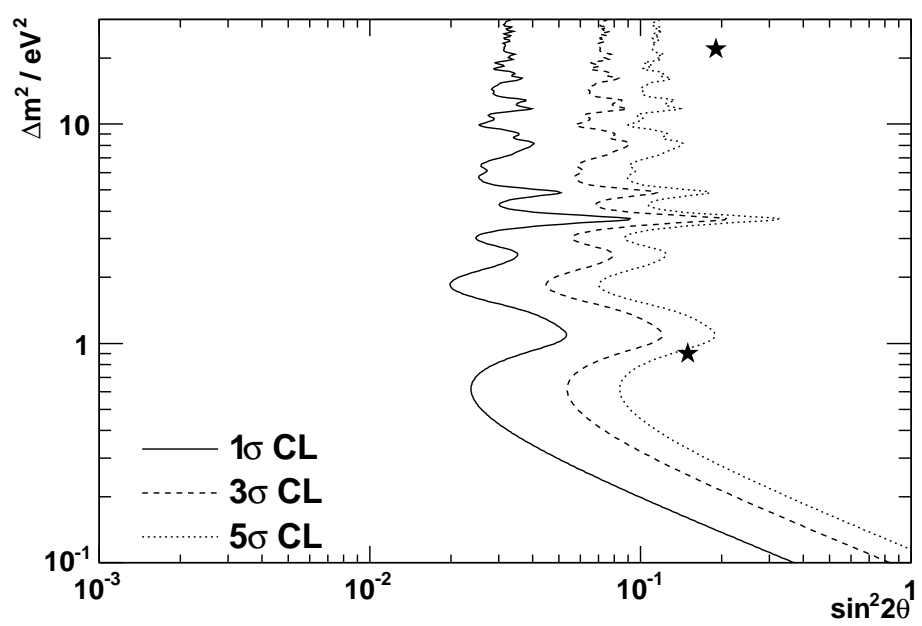

Figure 4: Active-Sterile neutrino oscillation sensitivity with the NC reaction $\nu_{\mathrm{x}}{ }^{12} \mathrm{C} \rightarrow$ $\nu_{\mathrm{x}}{ }^{12} \mathrm{C}^{*}(15.11)$ for SNS with two detectors, $5 \% \phi_{\nu_{\mu}} \times \sigma_{\mathrm{NC}}$ systematic errors, and three years of running. The two black stars correspond to the $(3+2)$ solutions (see text).

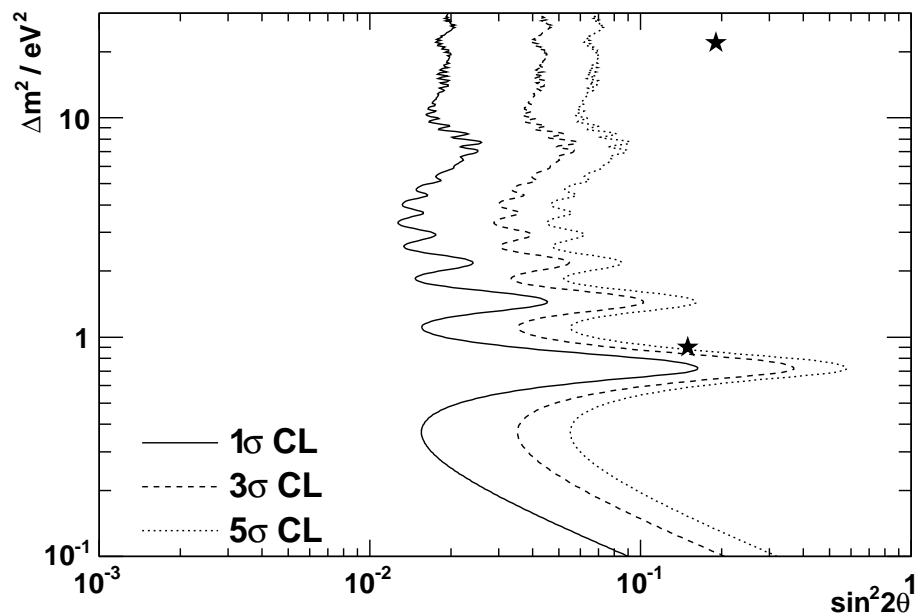

Figure 5: Active-Sterile neutrino oscillation sensitivity with the NC reaction $\nu_{\mathrm{x}}{ }^{12} \mathrm{C} \rightarrow$ $\nu_{\mathrm{x}}{ }^{12} \mathrm{C}^{*}(15.11)$ for FNAL PD with two detectors, 5\% $\phi_{\nu_{\mu}} \times \sigma_{\mathrm{NC}}$ systematic errors, and three years of running. The two black stars correspond to the $(3+2)$ solutions (see text). 


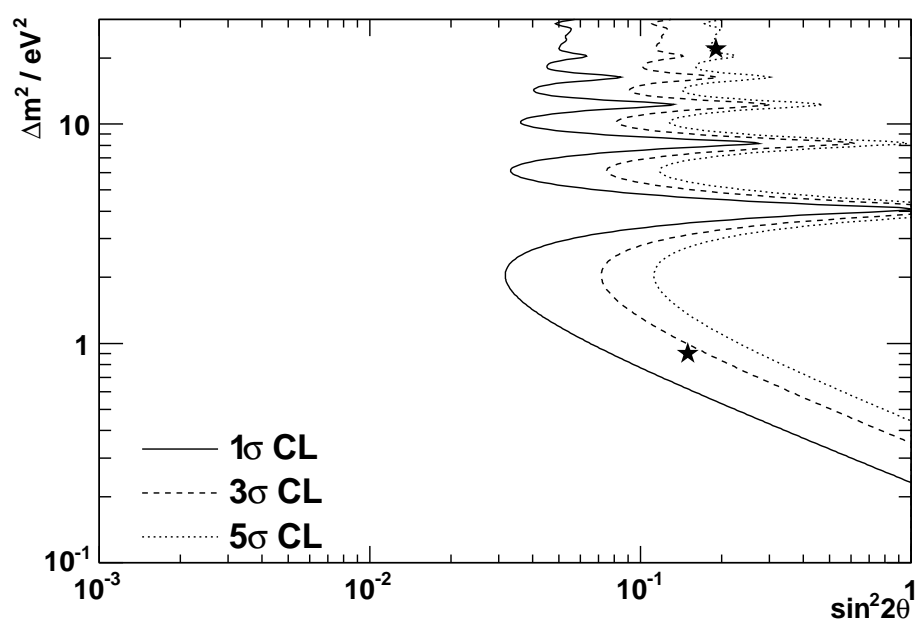

Figure 6: Active-Sterile neutrino oscillation sensitivity with the NC reaction $\nu_{\mathrm{x}}{ }^{12} \mathrm{C} \rightarrow$ $\nu_{\mathrm{x}}{ }^{12} \mathrm{C}^{*}(15.11)$ for SNS with a near detector, $5 \% \phi_{\nu_{\mu}} \times \sigma_{\mathrm{NC}}$ systematic errors, and three years of running. The two black stars correspond to the $(3+2)$ solutions (see text).

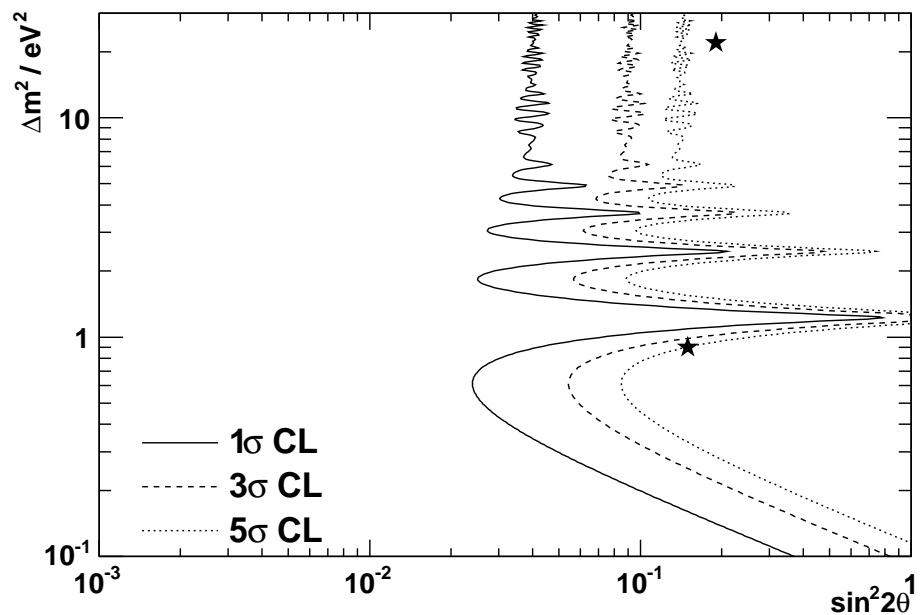

Figure 7: Active-Sterile neutrino oscillation sensitivity with the NC reaction $\nu_{\mathrm{x}}{ }^{12} \mathrm{C} \rightarrow$ $\nu_{\mathrm{x}}{ }^{12} \mathrm{C}^{*}(15.11)$ for SNS with a far detector, $5 \% \phi_{\nu_{\mu}} \times \sigma_{\mathrm{NC}}$ systematic errors, and three years of running. The two black stars correspond to the $(3+2)$ solutions (see text). 

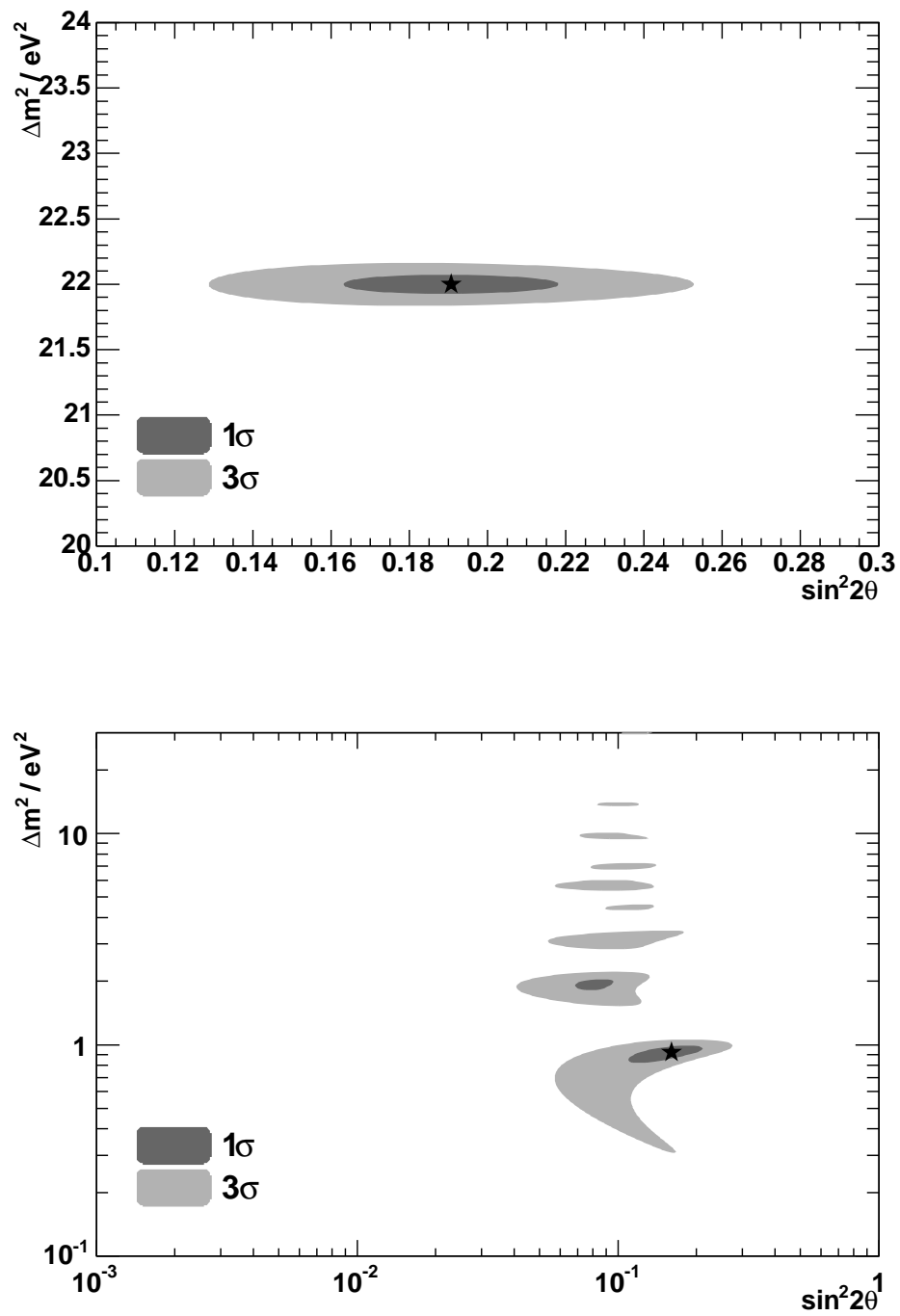

Figure 8: The $(3+2)$ active-sterile neutrino oscillation solution contours for SNS with two detectors, $5 \% \phi_{\nu_{\mu}} \times \sigma_{\mathrm{NC}}$ systematic errors, and three years of running. See text for definition of $\sin ^{2} 2 \theta$. 

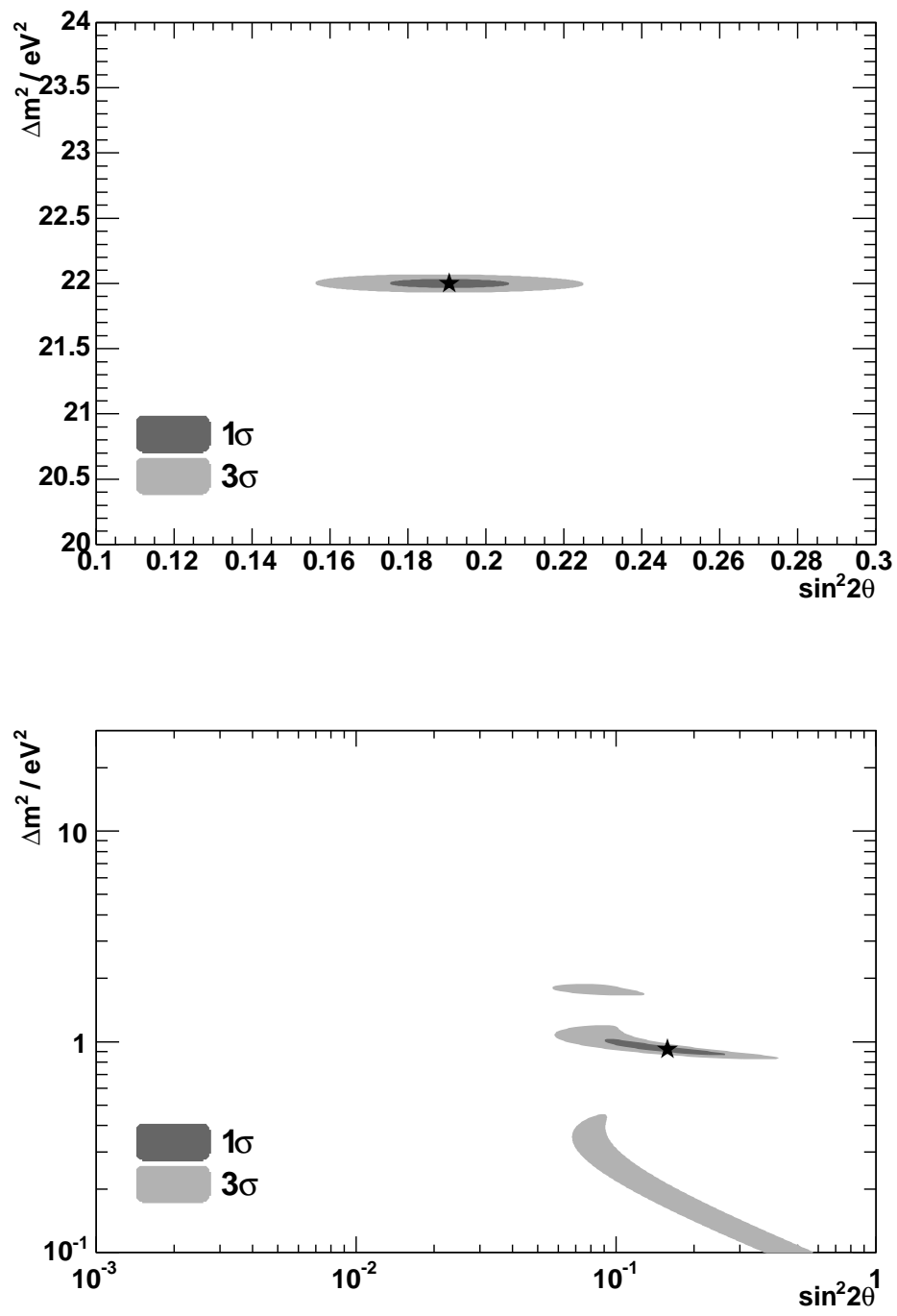

Figure 9: The $(3+2)$ active-sterile neutrino oscillation solution contours for FNAL with two detectors, $5 \% \phi_{\nu_{\mu}} \times \sigma_{\mathrm{NC}}$ systematic errors, and three years of running. See text for definition of $\sin ^{2} 2 \theta$. 